\title{
Significance of operating environment in condition monitoring of large civil structures
}

\author{
Sreenivas Alampalli \\ Structures Research, New York State Department of \\ Transportation, 1220 Washington Avenue, Albany, \\ NY 12232-0869, USA \\ Tel. +1 518457 5826; Fax: +1 5184577535 ; \\ E-mail: salampalli@gw.dot.state.ny.us
}

Received 9 November 1998

Revised 10 September 1999

Success of remote long-term condition monitoring of large civil structures and developing calibrated analytical models for damage detection, depend significantly on establishing accurate baseline signatures and their sensitivity. Most studies reported in the literature concentrated on the effect of structural damage on modal parameters without emphasis on reliability of modal parameters. Thus, a field bridge structure was studied for the significance of operating conditions in relation to baseline signatures. Results indicate that in practice, civil structures should be monitored for at least one full cycle of in-service environmental changes before establishing baselines for condition monitoring or calibrating finiteelement models. Boundary conditions deserve special attention.

Keywords: Condition monitoring of structures, damage detection, remote monitoring systems, bridges, reliability of test data, in-service bridge environment, bridge model calibration

\section{Introduction}

Remote monitoring of such large civil structures as bridges and dams, for condition monitoring and to develop analytical models has received considerable attention in the last decade. Several researchers concentrated their efforts on bridge structures, because their inspection relies largely on visual examination to detect deficiencies and to estimate bridge condition, in spite of lack of tools to document damage quantitatively.

Vibration monitoring for bridge diagnosis is one of these methods. Several researchers have investigated changes in modal parameters to simulated damage, using laboratory bridge models and field bridges [1,4]. Alampalli et al. [1] investigated sensitivity of measured modal parameters to simulated damage conditions by conducting repeated tests on a scaled model and an abandoned field bridge. They concluded that modal frequencies may be used to detect existence of damage in highway bridges. However, it is difficult to isolate damage locations. Such favorable conclusions led to development of analytical models calibrated using field data, and algorithms for damage detection and to interpret data from monitoring systems [6].

One major issue in developing condition monitoring systems is that experimental modal analysis, like other experimental techniques, produces variable results when repeated due to the influence of test environment, random variation associated with test and analysis procedures, and in-service environment. These variations may exceed the changes due to damage or deterioration, and thus can lead to incorrect conclusions. These effects consequently must be well understood before reliable use of modal-based damage detection through algorithms or analytical models. Very few studies are available addressing this issue, and other researchers have started to investigate this issue [1,2]. Alampalli et al. [1] studied the effects of instrumentation systems, test method, data reduction, and required manual operations on measured modal parameters of a scaled-bridge model and a field bridge. However, little attention has been given to sensitivity of modal parameters to in-service environment, which is critical for implementation in such practices as bridge or structural monitoring or inspection. This paper presents such results from experimental tests on a field bridge.

\section{Test structure}

A fracture-critical bridge with two steel girders, located over Mud Creek on Van Duesen Road in Claverack, NY, was field tested. This bridge was built in 1930 


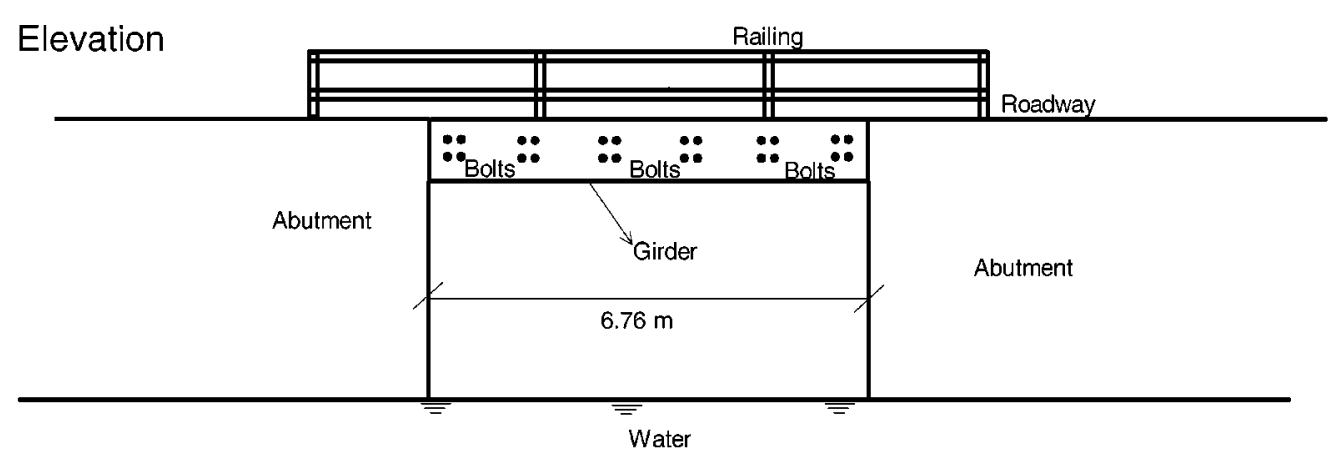

Typical section

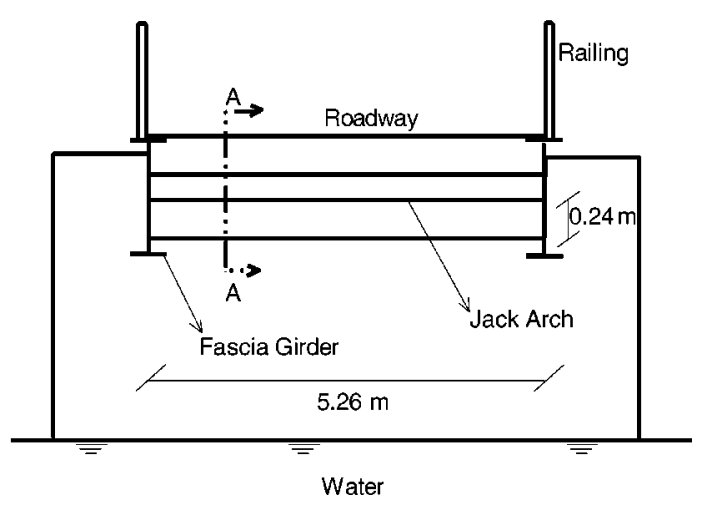

Section A-A

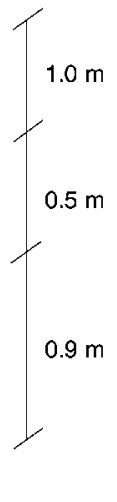

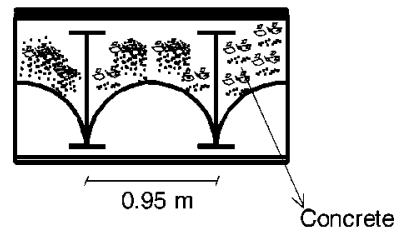

Fig. 1. Schematic of the bridge.

and closed to service in 1988 . It had two W18 $\times 64$ steel girders supporting floor beams and a reinforcedconcrete deck, as shown in Fig. 1. The floor beams were bolted to the main girders, which appeared to be embedded in the concrete abutments at both ends.

\section{Test method}

A general impact test setup [3] was used. An impulse force hammer (PCB Model 086B50) was used to excite the bridge, and the induced excitation was measured using a load cell (10 mV/lbf sensitivity) attached to the hammer tip. Structural responses were measured using an accelerometer (PCB Flexcel Model 336A04 with $100 \mathrm{mV} / \mathrm{g}$ sensitivity) fixed rigidly to the bridge's steel girders by a magnet. Only vertical vibration response perpendicular to the plane of the concrete deck was measured. A Tektronix Model 2630 dynamic signal analyzer obtained time-domain data, transfer functions, power spectra, and coherence functions [3].

A total of 54 measurement locations were chosen, such that behavior of the structure in the modes of in- terest could be represented [1]. The accelerometer location was chosen so that it was perceived and later verified not to be a modal node within the frequency range of interest. The hammer provided the excitation input at every test location, and the frequency response function (FRF) was obtained by measuring force input (of the impact hammer) and response output (of the stationary accelerometer). FRF was computed by the analyzer, which also performed signal digitization using appropriate antialiasing filters, by dividing the cross-power spectrum of output and input signals with auto-power spectrum of the input signal. An exponential window (filter) was used on the vibration response and the force window used on the hammer excitation. The purpose of a force window is to increase the signal to noise ratio in the force signal. The hammer impact pulse is short compared to time record length. Thus the force window consists of a rectangle of unity amplitude initially to preserve the pulse portion of the signal and then tapers quickly to zero to eliminate the noise [5]. Similarly, if the response of the structure has not decayed to zero by the end of the time record, the signal will produce leakage error in the estimated FRF. 
Thus the purpose of the exponential window is to add extra decay and artificially force the response to zero and avoid truncation error. Exponential window consists of a leading half cosine taper to unity, followed by an exponential decay with a suitable decay constant [5].

This test procedure was repeated for each point until a good coherence, above 0.95 in the frequency range of interest, was obtained and then the average of FRFs was stored as the input-output transfer function [3]. The coherence function indicated consistency of the obtained data, being 1.0 for perfect consistency and zero for no consistency. For all the data collected, almost perfect coherence values (greater than 0.95) were recorded in the frequency range of interest. Modal parameters were estimated using software from Structural Measurement Systems. In this paper, discussion is limited to the obtained modal frequencies.

\section{Influence of random variation}

In practice, all data acquisition systems and sensors can collect data to a limited accuracy. Test environment adds noise to measured structural responses. Required manual operations during data collection and subsequent analysis may introduce fluctuations in test results. All these random and uncontrollable factors contribute to non-systematic variation in test results. Establishing stable structural signatures and quantifying their variation thus may be critical for practical applications.

To quantify the effect of test environment on modal parameters, ten impact tests were conducted on the bridge to evaluate variation in measured modal frequencies. Data were obtained using a 0-200 Hz base band, with $0.125-\mathrm{Hz}$ frequency resolution. Table 1 gives the obtained modal frequencies and their statistical characteristics in terms of means, standard deviations, and coefficients of variation (i.e., standard deviations divided by the means). These results indicate that frequencies are estimated with relatively high consistency (within three times the employed frequency resolution), and thus can be used as fundamental structural signatures. This indicates that if the change in modal parameters is too small to be seen/recorded, in all likelihood no significant structural change will have occurred. They also demonstrate that if modal frequencies are to be used as structural signatures, changes in structural condition may not be detected if they result in small deviations, as those observed, from mean modal frequencies.
Table 1

\begin{tabular}{ccccc}
\multicolumn{3}{c}{ Random variation of modal frequencies for intact field bridge } \\
\hline Test & Air & \multicolumn{3}{c}{ Modal frequencies $(\mathrm{Hz})$} \\
\cline { 2 - 5 } number & temp. & Mode & Mode & Mode \\
& $\left({ }^{\circ} \mathrm{F}\right)$ & 1 & 2 & 3 \\
\hline 1 & 57 & 10.832 & 20.176 & 36.848 \\
2 & 45 & 10.875 & 20.275 & 36.916 \\
3 & 44 & 10.974 & 20.230 & 36.920 \\
4 & 48 & 10.905 & 20.145 & 36.777 \\
5 & 48 & 10.880 & 20.290 & 36.776 \\
6 & 49 & 10.933 & 20.266 & 36.750 \\
7 & 46 & 10.901 & 20.250 & 36.752 \\
8 & 45 & 10.858 & 20.183 & 36.621 \\
9 & 42 & 10.886 & 20.157 & 36.728 \\
10 & 42 & 10.906 & 20.150 & 36.833 \\
Range & $57-42$ & $10.974-$ & $20.290-$ & $36.920-$ \\
& & 10.832 & 20.150 & 36.621 \\
Mean & 46.6 & 10.895 & 20.212 & 36.792 \\
STD & 4.152 & 0.037 & 0.053 & 0.0859 \\
COV $(\%)$ & 8.901 & 0.343 & 0.263 & 0.233 \\
\hline & & & &
\end{tabular}

\section{Effect of in-service conditions}

Sensitivity of baseline signature was established as described in the previous section. Effects of in-service environment on modal parameters for condition monitoring or calibrating analytical models for damage detection are now described.

Weather conditions in upstate New York, where the test bridge is located, vary greatly by season and thus the field bridge experiences extreme cold and hot temperatures. It was decided to test the structure during below- and above-freezing temperatures to investigate effects of in-service temperatures on structural signatures. When these tests were conducted, the structure was in a seriously damaged state and the data here correspond to a case where both bridge girders had been cut through the entire flange width and thickness. Tests were repeated 16 times on 13 different days.

Tests were identical to those previously described, except that temperature in the field was near freezing during winter. After data were obtained, a simple statistical analysis was conducted to obtain confidence intervals, assuming both temperature and simulated damage to be influencing factors, and adjusting means for temperature effects. Confidence intervals were also estimated assuming the simulated damage to be the only influencing factor. These results showed that temperature indeed was influencing baseline signatures (i.e., modal frequencies). Careful examination of test results indicated that it would be appropriate to split the data 
into two groups based on field air temperature. Because measured temperatures under the bridge were 5 to $6^{\circ} \mathrm{F}$ lower than air temperature, $38^{\circ} \mathrm{F}$ was selected as the cutoff value.

Results of modal tests for above-freezing temperatures are shown in Table 2, and were then statistically analyzed to estimate confidence intervals, assuming both temperature and simulated damage to be influencing factors, and adjusting means for temperature effects. Confidence intervals were also estimated assuming the simulated damage to be the only influencing factor. Based on this statistical analysis, it was concluded that above-freezing temperature had no practical or significant effect.

Comparison of modal results obtained, at abovefreezing temperatures, from damaged state (Table 2 and Fig. 2) with intact state (Table 1 and Fig. 2) show that the variation of modal frequencies for damaged state is higher. However, summarized statistics in the tables also show that modal frequencies have changed significantly from the intact state. Maximum values for damaged-state frequencies are noticeably lower than corresponding minimum frequencies in the intact state for all three modes. This significant difference indicates that a change has occurred in the structure's condition. The observed drop in frequencies is also consistent with the fact that modal frequencies of a structure decrease with loss of stiffness. From Tables 1 and 2 , not even knowing the structure's condition, one may

Table 2

Variation of modal frequencies of field bridge, with simulated damage, at above-freezing temperatures

\begin{tabular}{ccccc}
\hline Test & Air & \multicolumn{3}{c}{ Modal frequencies $(\mathrm{Hz})$} \\
\cline { 2 - 5 } number & $\begin{array}{c}\text { temp. } \\
\left({ }^{\circ} \mathrm{F}\right)\end{array}$ & 1 & 2 & 3 \\
\hline 1 & 59 & 10.195 & 19.794 & 35.357 \\
2 & 57 & 10.106 & 19.762 & 35.289 \\
3 & 62 & 10.038 & 19.713 & 35.140 \\
4 & 60 & 9.940 & 19.683 & 34.974 \\
5 & 61 & 9.977 & 19.791 & 35.629 \\
6 & 53 & 10.055 & 19.774 & 36.058 \\
7 & 69 & 9.953 & 19.780 & 35.395 \\
8 & 74 & 10.001 & 19.715 & 35.059 \\
9 & 50 & 9.876 & 19.369 & 34.771 \\
10 & 64 & 9.856 & 19.496 & 34.739 \\
Range & $74-50$ & $10.195-$ & $19.794-$ & $36.058-$ \\
& & 9.856 & 19.369 & 34.739 \\
Mean & 60.85 & 10.000 & 19.688 & 35.241 \\
STD & 6.693 & 0.098 & 0.135 & 0.380 \\
COV $(\%)$ & 11.000 & 0.981 & 0.687 & 1.079 \\
\hline
\end{tabular}

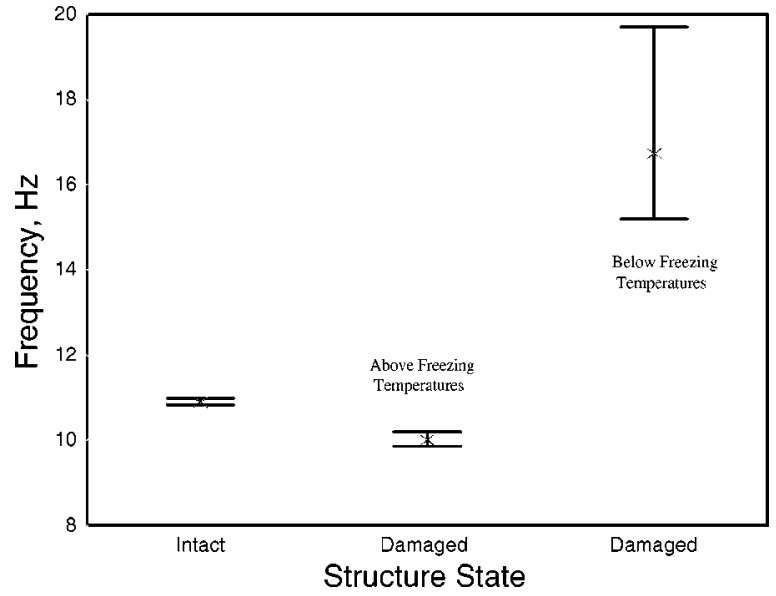

Fig. 2. Effect of in-service environment on the natural frequencies and damage detection.

easily conclude with high certainty that the structure has suffered stiffness loss due to the inflicted damage.

Results of modal tests for below-freezing temperatures are given in Table 3. Comparison of the intact frequencies with those in Table 3 for below-freezing temperatures suggests an alternative conclusion - that is, structural stiffness has increased significantly, because these frequencies are higher than the corresponding intact bridge frequencies in Table 1 (see Fig. 2).

\section{Discussion}

Careful study of structural plans and on-site inspection indicated frozen supports as a probable cause for this unusual condition. Freezing of accumulated moisture (from a creek beneath the bridge) at the supports caused partial fixity at the ends of the embedded girders, and resulted in the apparent increase in modal frequencies.

The ratio between natural frequencies of a fixed and simply supported beam can be estimated using the theory of vibration for continuous systems. This ratio ranges between 1.4 and 2.3 for the first three modes, depending on loading (uniform or concentrated) and consideration of the beam's self-weight [7]. Similar ratios for mean frequencies in Tables 2 and 3 were consistent with theoretical values and ranged from 1.4 to 1.7 , supporting the conclusion of partial-fixity of the structure due to frozen supports.

Although the observed seasonal changes were important for better understanding of the tested structure (see Fig. 2), these changes (in this case due to environment or partial fixity of bearings) may be of little im- 
Table 3

Variation of modal frequencies of field bridge, with simulated damage, at below-freezing temperatures

\begin{tabular}{|c|c|c|c|c|}
\hline \multirow{3}{*}{$\begin{array}{c}\text { Test } \\
\text { number }\end{array}$} & \multirow{3}{*}{$\begin{array}{c}\text { Air } \\
\text { temp. } \\
\left({ }^{\circ} \mathrm{F}\right)\end{array}$} & \multicolumn{3}{|c|}{ Modal frequencies (Hz) } \\
\hline & & Mode & Mode & Mode \\
\hline & & 1 & 2 & 3 \\
\hline 1 & 36 & 19.706 & 32.316 & 66.258 \\
\hline 2 & 36 & 17.201 & 28.102 & 56.214 \\
\hline 3 & 31 & 15.586 & 26.979 & 52.391 \\
\hline 4 & 30 & 15.198 & 25.964 & 52.424 \\
\hline 5 & 34 & 16.641 & 28.677 & 57.425 \\
\hline 6 & 36 & 16.032 & 27.467 & 48.670 \\
\hline Range & $36-30$ & $\begin{array}{c}19.706- \\
15.198\end{array}$ & $\begin{array}{c}32.316- \\
25.964\end{array}$ & $\begin{array}{c}66.258- \\
48.670\end{array}$ \\
\hline Mean & 33.75 & 16.727 & 28.251 & 55.564 \\
\hline STD & 2.41 & 1.485 & 2.008 & 5.563 \\
\hline $\operatorname{COV}(\%)$ & 7.14 & 8.877 & 7.108 & 10.012 \\
\hline
\end{tabular}

Note: Since, measured temperatures under the bridge were 5 to $6^{\circ} \mathrm{F}$ lower than air temperature, $38^{\circ} \mathrm{F}$ was selected as belowfreezing temperature.

portance to a bridge owner, and would not generally be alarming unless they compromise structural integrity. However, for a condition monitoring system and damage assessment, use of such data without proper understanding, could result in significant misdirection of effort and resources. If the damaged bridge data were recorded at a temperature corresponding to limited fixity and were used to calibrate the damage assessment algorithm or analytical models, an inaccurate conclusion could be drawn that damage did not influence the structure's frequencies (see Fig. 2). Thus, if the base line of the intact structure was established during summer and the damage occurred during winter, conclusions might have been inaccurate. This shows the influence of in-service structural conditions that should be properly accounted for when developing condition monitoring systems, calibrated models, and damage detection algorithms.

\section{Conclusions}

Sensitivity of modal parameters is inherent to testing and analysis methods, as well as the structure's inservice condition. Damage assessment could be significantly influenced by these factors, and they thus should be studied carefully before reaching any con- clusions. In-service/operating environment can significantly influence modal parameters, without compromising strength or safety. These are entirely dependent on the structure and its environment, and thus are unique for each structure. They should be well understood before attempting damage assessment by continuous monitoring of any structure. Bridges should be monitored for at least one full cycle of in-service conditions, before establishing warning triggers for condition monitoring or using damage assessment algorithms, and one must use great care in interpreting their output before adopting automated warning/alarm systems.

\section{Acknowledgments}

Many New York State Department of Transportation personnel contributed to success of this study. The views expressed are those of the author and not necessarily of the New York State Department of Transportation. This work was partially funded by the Federal Highway Administration, U.S. Department of Transportation.

\section{References}

[1] S. Alampalli, G. Fu and E.W. Dillon, Measured bridge vibration for detection of structural damage. Research report 165 , Transportation R\&D Bureau, New York State Department of Transportation, 1995.

[2] A. Bensalem et al., Nondestructive testing for condition based maintenance of arch bridges, in: Proc. 8th Int. Congress on Condition Monitoring and Diagnostic Engineering Management, Kingston, Ontario, Canada, 2, 1995.

[3] D.J. Ewins, Modal Testing: Theory and Practice, Wiley, New York, 1984.

[4] D.F. Mazurek and J.T. DeWolf, Experimental study of bridge monitoring technique, ASCE J. of the Structural Division 116(9) (1990)

[5] R.C. Sohaney and J.M. Nieters, Proper use of weighting functions for impact testing, in: Proc. 3rd Int. Modal Analysis Conf., Orlando, Florida, 1985.

[6] T. Tokspy and A.E. Aktan, Bridge condition assessment by modal flexibility, Experimental Mechanics 34 (1994).

[7] W.C. Young, Roark's Formulas for Stress and Strain, McGrawHill, New York, 1989. 

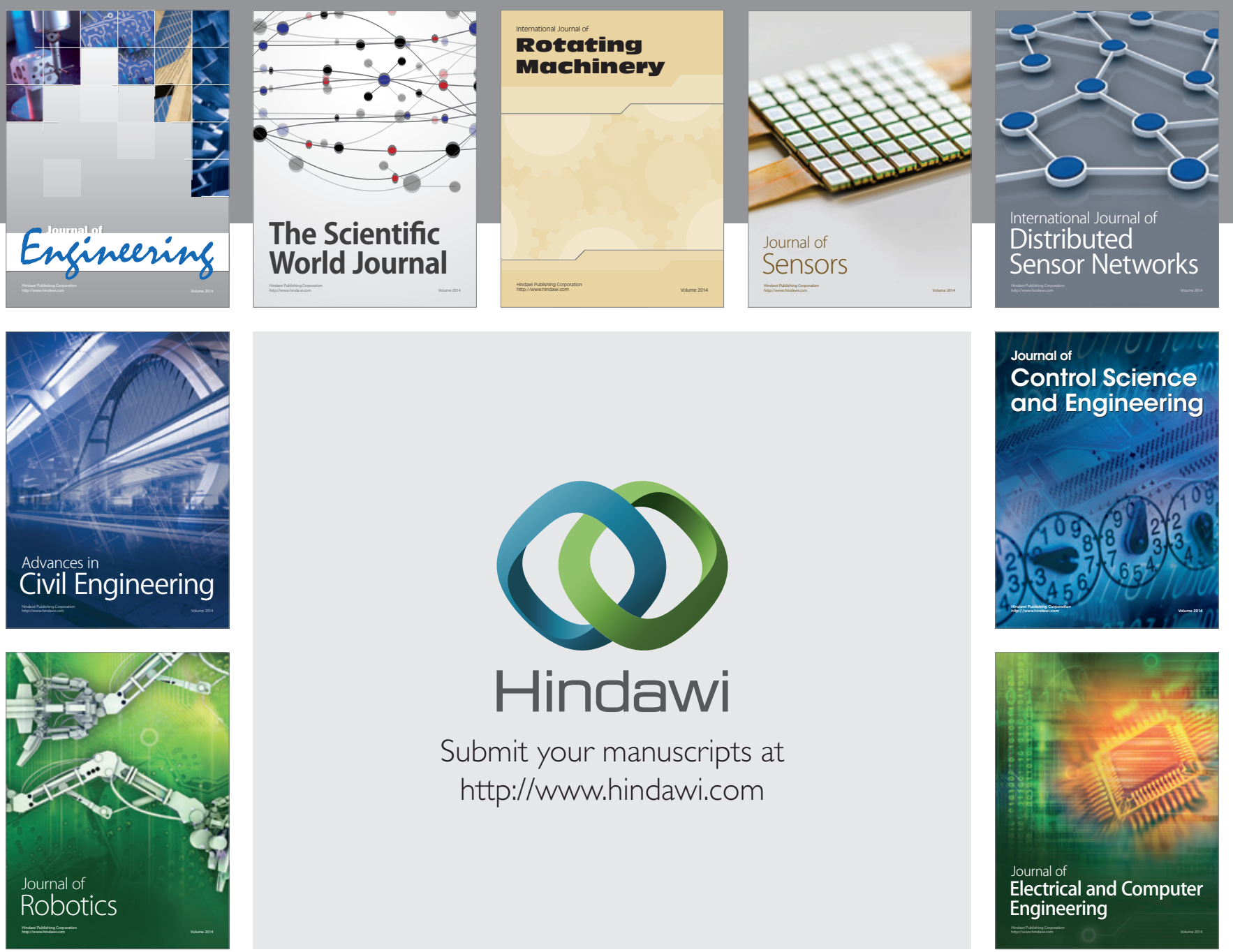

Submit your manuscripts at

http://www.hindawi.com
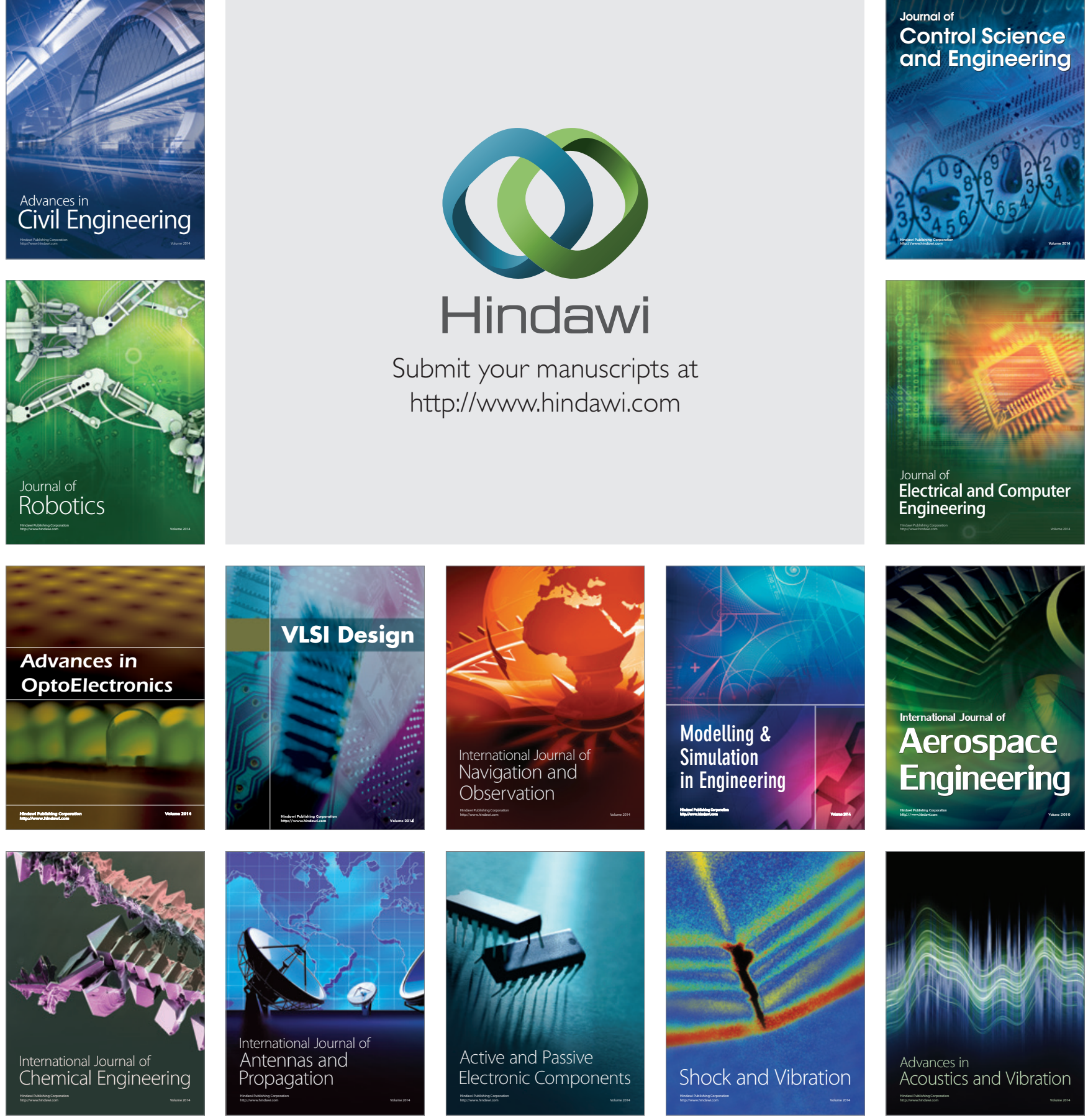